\title{
PLANTAS EN LA OLLA: ANÁLISIS DE MICRORRESTOS VEGETALES APLICADO A CERÁMICA DEL SITIO ARQUEOLÓGICO LAS MARÍAS, PROVINCIA DE BUENOS AIRES
}

\section{PLANTS IN THE COOKING POT: ANALYSIS OF MICROREMAINS VEGETABLES APPLIED TO CERAMICS FROM THE LAS MARÍAS ARCHAEOLOGICAL SITE, BUENOS AIRES PROVINCE}

Melisa A. Auge', Diego F. Andreoni², M. Mercedes Pérez Meroni³, M. Clara Paleo ${ }^{4}$

${ }^{1}$ Laboratorio de Análisis Cerámico, FCNyM-UNLP. Calle 64 n³ CP 1900, La Plata, Buenos Aires, Argentina. Becaria doctoral CONICET. Email: augemelisa@gmail.com

2 Laboratorio 129, División de Arqueología, FCNyM-UNLP. Av. 60 s/n CP 1900, La Plata, Buenos Aires, Argentina. CONICET. Email: andreondieg@hotmail.com

${ }^{3}$ Laboratorio de Análisis Cerámico, FCNyM-UNLP. Calle 64 nº3 CP 1900, La Plata, Buenos Aires, Argentina. Email: mperezmeroni@yahoo.com.ar

${ }^{4}$ Laboratorio de Análisis Cerámico, FCNyM-UNLP. Calle 64 n³ CP 1900, La Plata, Buenos Aires, Argentina. Email: mcpaleo@fcnym.unlp.edu.ar

Presentado: 29/10/2020

Aceptado: 26/02/2021

\section{Resumen}

En este trabajo presentamos los resultados del análisis de microrrestos vegetales recuperados en los diferentes tipos morfo-funcionales de cerámica del sitio Las Marías (partido de Magdalena, Buenos Aires). Para su abordaje e interpretación, incluimos el estudio tanto de fuentes documentales como de una colección de referencia de plantas presentes en la zona de interés. Esta investigación reveló las primeras evidencias directas de uso de plantas domesticadas para la zona de estudio en coexistencia con otras silvestres locales. También se identificaron distintas estrategias para el aprovechamiento de vegetales que se corresponderían a diferentes usos de los tipos alfareros presentes en el sitio.

Palabras clave: manejo del entorno, procesamiento de vegetales, plantas silvestres, plantas domesticadas

\section{Abstract}

In this paper, we present the results of the analysis of vegetable microremains recovered in different morpho-functional types of ceramics from Las Marías site (Magdalena, Buenos Aires). For its approach and interpretation, we include the study of both documentary sources and a reference collection of plants present in the study area. This work revealed the first direct evidence of the use of domesticated plants for the study 
area in coexistence with other local wild plants. Moreover, we identify different strategies for the usage of vegetables that might correspond to the diverse use of the pottery types present on the site.

Keywords: environment management, processing plants, wild plants, domestic plants

\section{Introducción}

En Argentina, las investigaciones arqueológicas que incluyen análisis de microrrestos vegetales adquirieron mayor preponderancia y aumentaron considerablemente en los últimos años a lo largo de la Cuenca del Plata (Zucol y Loponte 2008; Pérez Meroni et al. 2010; Bonomo et al. 2011; Cornero y Rangone 2015; Colobig et al. 2016; Colobig y Ottalagano 2016; Auge et al. 2018; entre otros). El mismo proceso se ve reflejado en las producciones científicas sobre las costas uruguayas (ie. Beovide 2011; Beovide y Campos 2015). La sistematización de estos trabajos incentivó la identificación de una variedad de taxones presentes en el registro arqueológico en zonas donde la preservación de macrorrestos vegetales es escasa.

En este contexto, comenzaron a desarrollarse estudios centrados en la interrelación entre las plantas y grupos humanos de época prehispánica asentados en la porción meridional del litoral rioplatense, zona distintiva por presentar una gran riqueza biocultural, hoy declarada y conocida como Reserva Mundial de Biosfera Parque Costero del Sur (UNESCO). La trayectoria de nuestras investigaciones enfatiza y enlaza los resultados de la identificación taxonómica con la información procedente de otros registros, camino que nos permite conocer con mayor profundidad los contextos socioculturales en los que interactuaban las personas, la alfarería y las plantas en el pasado.
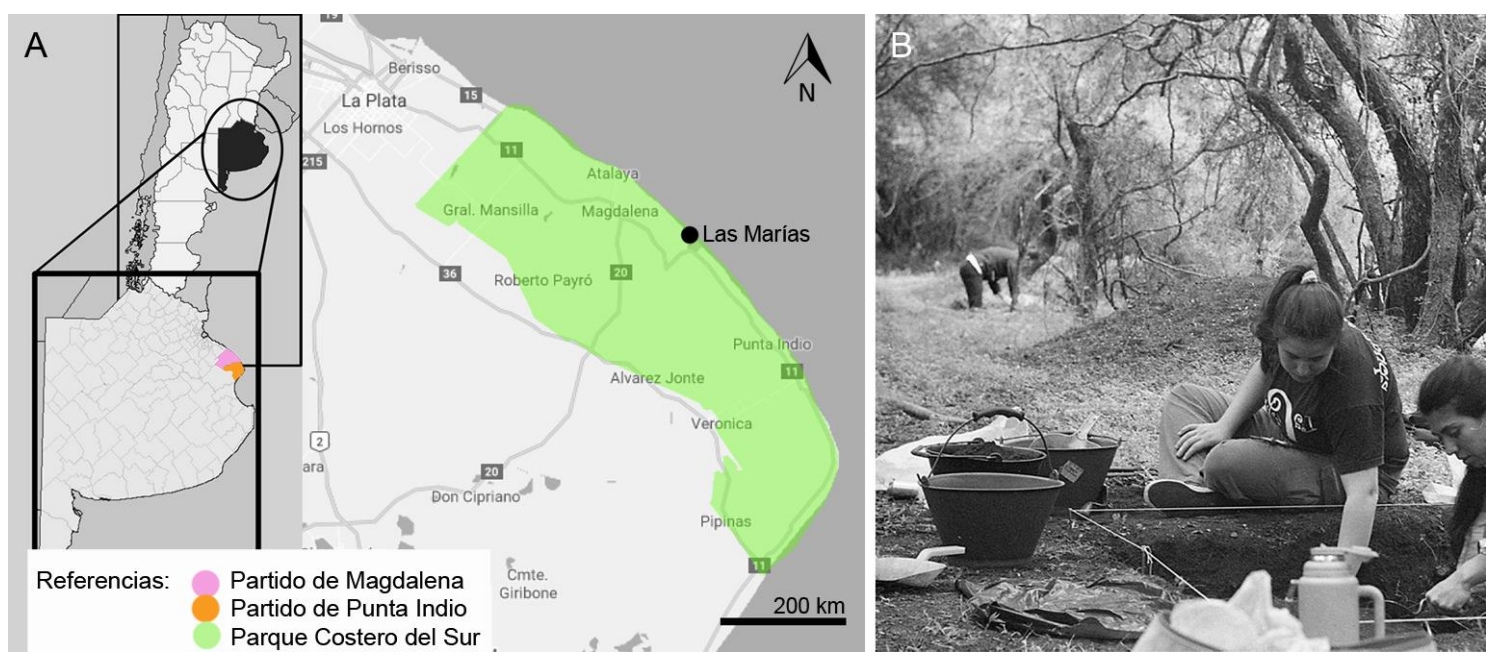

Figura 1. a) ubicación del sitio arqueológico Las Marías, emplazado dentro del Parque Costero del Sur. b) excavación de Las Marías donde se observa la presencia del bosque de tala (Foto: M.

Auge, 2014). 
El objetivo de este trabajo es presentar los resultados del análisis de microrrestos vegetales recuperados en los diferentes tipos morfo-funcionales de cerámica del sitio Las Marías, ubicado en el actual partido de Magdalena (Magdalena, provincia de Buenos Aires) (Figura 1). Estos estudios aportan información sobre las prácticas asociadas al manejo y aprovechamiento de plantas por las poblaciones humanas que incluyen los conocimientos y creencias involucrados en la relación de los seres humanos y su ambiente (Pochettino y Lema 2008). De esta forma, nos introducen en la discusión sobre el tipo de prácticas de manejo del entorno vegetal que habrían implementado estos grupos, y la importancia de estos a nivel regional.

\section{El sitio Las Marías: estado actual de conocimiento}

En el partido de Magdalena, a $1 \mathrm{~km}$ de la costa rioplatense se localiza el sitio Las Marías (Figura 1). Se trata de un asentamiento a cielo abierto datado en $1820 \pm$ 50 años AP (Pogonias cromis) y $1590 \pm 40$ años AP (Lama guanicoe) (Paleo et al. 2002, Paleo y Pérez Meroni 2007). Se emplaza en zonas elevadas producidas por la acumulación de sedimentos conchiles depositados por ingresiones marinas, que se encuentran dispuestos en forma paralela a subparalela a las costas del Río de la Plata y proporcionaron el sustrato necesario para el desarrollo del "bosque de tala" (Paleo y Pérez Meroni 2004, 2007). Esta formación boscosa es una de las características florísticas más destacables del área y se compone principalmente por Celtis tala Gillies ex Planch. (tala), Scutia buxifolia Reissek (coronillo), Jodina rhombifolia (Hook. \& Arn.) Reissek (sombra de toro), por mencionar las más abundantes (Delucchi y Torres Robles 2009). La ocupación humana habría encontrado en el bosque refugio y protección que, junto a ambientes circundantes asociados a pastizales, humedales y el río, ofrecen una variada gama de recursos. Se propuso que las poblaciones que ocuparon Las Marías tenían una economía basada en la caza, pesca y recolección de gran cantidad de especies locales. En el registro arqueofaunístico y asociados fundamentalmente a actividades antrópicas se destacan los peces (principalmente Pogonias cromis y Pterodoras granulosus), aves (ie. Rhea americana) y mamíferos de diversos tamaños como cérvidos (Ozotoceros bezoarticus y Blastocerus dichotomus), carnívoros terrestres (Puma concolor) y marinos (Arctocephalus australis, Otaria flavescens), y roedores caviomorfos (ie. Cavia aperea, Ctenomys talarum). Se evidencia el consumo integral de muchas especies, incluyendo el aprovechamiento tecnológico para confeccionar instrumentos óseos como alisadores, puntas y punzones (Day Pilaría 2018). El hallazgo de elementos alóctonos como material lítico y restos óseos de Lama guanicoe permitieron proponer que estos grupos podrían haber participado en redes de interacción a escala regional (Paleo y Pérez Meroni 2005-2006).

La cerámica constituye el registro más abundante y se halla con un alto grado de fragmentación. Desde hace 30 años, el equipo del Laboratorio de Análisis Cerámico (LAC), de la Facultad de Ciencias Naturales y Museo (UNLP), implementó diferentes 
estudios arqueométricos para el abordaje de la cerámica: análisis de la decoración, difractometrías de rayos $X$, cortes delgados, análisis de porosidad, estudios experimentales y análisis químicos de residuos orgánicos. La tarea de remontaje permitió reconocer al menos tres tipos básicos de formas, tamaño y funciones diferenciadas (Figura 2): 1) contenedores de procesamiento y/o servicio, 2) contenedores de almacenaje y 3) instrumentos de transferencia, conocidas como alfarerías tubulares (Paleo y Pérez Meroni 2005-2006; 2008; Ghiani Echenique y León 2014; Ghiani Echenique et al. 2017).
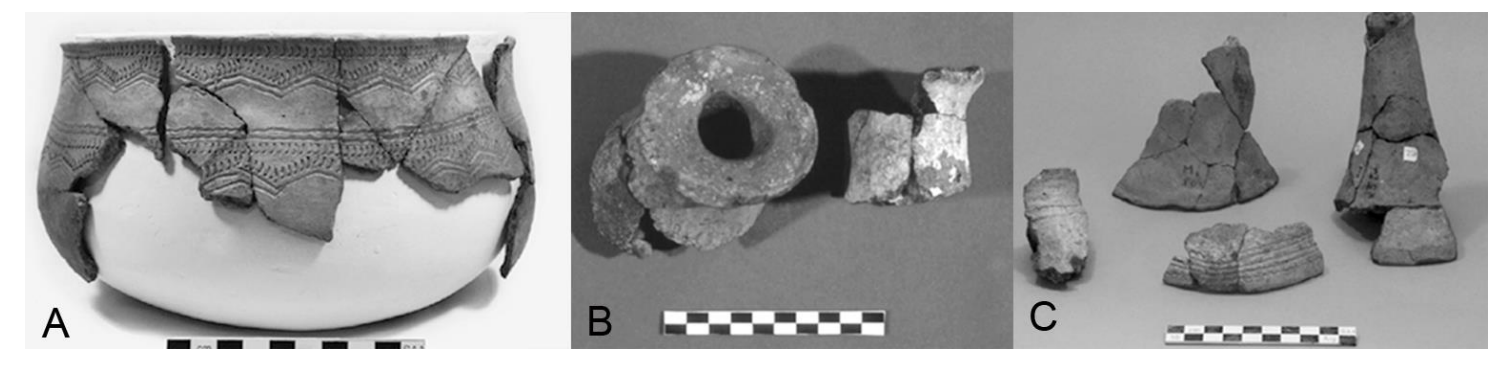

Figura 2. a) Contenedores de procesamiento y/o servicio, b) Contenedores de almacenaje y c) Instrumentos de transferencia.

Entre la multiplicidad de hallazgos que componen el registro arqueológico de Las Marías, los vegetales son los que presentan menos visibilidad en forma macroscópica. Los carbones no cuentan con condiciones de preservación favorables debido a la humedad y el pH del suelo sumado a la importante actividad biótica. Cabe destacar que la conservación del material vegetal se relaciona también con características intrínsecas de cada taxón y con factores antrópicos, como la selección diferencial por parte de las poblaciones humanas de determinadas especies y órganos vegetales, los tipos de procesamientos realizados, patrones culturales de descarte y depositación (Ford 1979).

Los primeros análisis arqueobotánicos de la zona se realizaron sobre fragmentos de contenedores de procesamiento con marcas de uso en forma de adherencias y/o hollín (Pérez Meroni et al. 2010; Lema et al. 2010). Estos estudios identificaron células pétreas y almidones de Celtis tala, y cristales y almidones de Jodina rhombifolia. Algunos granos de almidón presentaron distintos grados de alteración, como baja a nula birrefringencia, y fisuras en la zona del hilio. Por otro lado, se hallaron algunas estructuras ubicuas en el reino vegetal (como granos de almidón esféricos). Estas tareas aportaron evidencia directa del uso de vegetales, permitieron identificar procesos de manejo del entorno y distintas prácticas de procesamiento y consumo de plantas de la rivera rioplatense. En este sentido, en la presente contribución continuamos con esta línea de trabajo y pretendemos profundizar a futuro con su aplicación a nuevos registros arqueológicos de la costa del Río de la Plata. 


\section{Materiales y métodos}

\section{Colección de referencia}

Con el propósito de generar material comparativo para el análisis de microrrestos vegetales elaboramos una colección de referencia de especies relevantes. Los taxones que conforman la colección fueron seleccionados en función de las especies propias del bosque de tala mencionadas en distintas fuentes documentales con algún uso o procesamiento que podría vincularse al registro cerámico que aquí analizamos (como alimentos, bebidas, medicinas). También se incluyeron plantas domesticadas mencionadas para regiones vecinas al área de estudio. Para tal fin, analizamos fuentes de los siglos XVI al XIX (Ramírez 2007 [1528]; Lopes de Sousa 1927 [1530-1532]; Schmidel 1903 [1534-1554]; Vargas Machuca 1892 [1599]; Paucke 2010 [1749-1767] y Hieronymus 1882), y trabajos científicos de los siglos XX y XXI (Lévi-Strauss 1952; Arenas 2003; Keller 2007, 2008; Pochettino et al. 2016; Doumecq 2019). Para su abordaje tuvimos en cuenta las estrategias metodológicas propuestas por Nacuzzi (2002), entre las que se propone el análisis textual y contextual de los documentos.

La colección de referencia comprende, además de la caracterización de las estructuras en estado fresco, la descripción de modificaciones producidas por distintos tipos de procesamiento experimental (machacado, tostado, horneado, hervido y fermentado) observadas según el taxón. Para la descripción de las estructuras micrográficas utilizamos los parámetros consensuados en el International Code for Starch Nomenclature (ICSN 2011) y el International Code for Phytolith Nomenclature 2.0 (Neumann et al. 2019). En el caso de plantas cultivadas y/o ampliamente estudiadas recurrimos a bibliografía especializada (ie. Winton y Winton 1932; Reichert 1913). En esta oportunidad tendremos en cuenta el conjunto de microrrestos hallados en las muestras arqueológicas, ya que estas forman parte del contexto relacional que estamos analizando y son imprescindibles para el alcance de una determinación taxonómica, así como para el reconocimiento de prácticas de procesamiento.

\section{Material arqueológico}

Seleccionamos para este trabajo trece fragmentos cerámicos correspondientes a las tres categorías morfo-funcionales que rotulamos como " $\mathrm{B}$ ". Se trata de nueve fragmentos de contenedores de procesamiento y/o servicio, dos de contenedores de almacenaje y dos de instrumentos de transferencia. La diferencia en la cantidad de elementos seleccionados para cada grupo cerámico se corresponde con la frecuencia de estos en el registro arqueológico. Los fragmentos de contenedores de procesamiento y/o servicio superan en número a la cantidad de los otros tipos morfofuncionales, de la misma manera que presenta una mayor diversidad de formas y tamaños. 
Para la recuperación de microrrestos vegetales raspamos los fragmentos cerámicos en su cara interna y externa con instrumental metálico esterilizado a fuego. Con el fin de evitar la contaminación de la muestra se trabajó en ambiente cerrado y con superficies de trabajo limpias. Escogimos como medio de montaje aceite de inmersión ya que permite la observación de las estructuras vegetales en sus tres dimensiones. Realizamos una observación directa, sin tratamientos químicos previos para preservar la totalidad de las estructuras vegetales, en Microscopio Óptico Leica MDLM (MO) bajo aumentos de 20x y 50x, con luz normal y polarizada, y fotografiamos con cámara Toup CamTM modelo UCMOSO5100KPA.

Las condiciones edáficas de la región de estudio requieren que determinan que los materiales arqueológicos sean sometidos a tareas de acondicionamiento para su análisis e identificación. La reciente apertura de nuevos sectores de excavación en el sitio Las Marías nos permitió incluir tres muestras testigo previo a actividades de lavado, siglado y sistematización. De esta forma, analizamos tres fragmentos de contenedores de procesamiento y/o servicio siguiendo el protocolo de recuperación de microrrestos vegetales mencionado anteriormente. Estas muestras control, que agrupamos bajo la categoría "M", elevan la cantidad de fragmentos analizados a un total de dieciséis.

En los casos en los que registramos costras carbonizadas adheridas a bases de contenedores de procesamiento, para su observación en $\mathrm{MO}$ las tratamos con ácido láctico durante doce días y disgregamos mecánicamente con un agitador automático (tres sesiones de veinte minutos cada una). En estas muestras, el ácido láctico también sirvió como medio de montaje. Este protocolo, testeado de forma favorable en residuos carbonizados recuperados en pipas (Lema et al. 2015; Auge et al. 2018), permite aclarar y mejorar la visión al MO del material, sin perder la diversidad de estructuras vegetales.

\section{Resultados}

\section{Material de referencia}

La información de las plantas relevadas en las fuentes documentales y trabajos etnobotánicos que incorporamos a la colección de referencia fue sistematizada en la Tabla 1. Se trata de vegetales que tienen elementos con parámetros comparables a los observados en las muestras arqueológicas, algunas son taxones de crecimiento espontáneo en la zona de estudio y otras son especies domesticadas de circulación macrorregional. En la misma, también se consignan los tipos de procesamiento asociados a distintos órganos vegetales, así como usos y prácticas sociales en los que intervenían estas plantas. Cabe aclarar que Lopes de Sousa [1530-1532] en su viaje hace contacto con nativos en la zona de la desembocadura del río Santa Lucía y delta del Paraná, con quienes realiza intercambios de pescado, venado y "oveja", pero no incluye vegetales domesticados como sí lo hacen otros viajeros que transitan la región 
paranaense (Ramírez [1528] y de Schmidel [1534-1554]). Sin embargo menciona que en períodos de hambruna "nam comemos senam enam ervas cozidas" (Lopes de Sousa 1927 [1530-1532]: 318). De la misma forma, distintos exploradores señalan que se valieron de hierbas, posiblemente silvestres, para alimentarse cuando no había otros recursos (Ramírez [1528]; Lopes de Sousa [1530-1532]; Schmidel [1534-1554]).

En la Tabla 1 se puede observar una serie de usos asociados a órganos que tienen su correlato en el registro arqueológico aquí presentado. Sin embargo, en las fuentes analizadas también se mencionan otras formas de relacionarse con las mismas plantas en función de otras partes y propiedades de estas. Es el caso del uso de semillas y hojas de Manihot esculenta como alimento y medicina (Hieronymus 1882; Keller 2007, 2008). Árboles y arbustos de crecimiento espontáneo como Celtis tala presentan múltiples evidencias de uso como combustible (Pochettino et al. 2016; Doumecq 2019). Las hojas y corteza de Jodina rhombifolia son utilizadas como medicinales, y su tallo en la fabricación de una variedad de instrumentos (Hieronymus 1882; Pchettino et al. 2016). Según la creencia popular, este arbusto es protector, no le afectarían las inclemencias del tiempo como sequías, vientos o rayos, y el comportamiento de sus hojas anuncia la presencia de lluvias (Pochettino et al. 2016). Los órganos foliares de Manihot grahami (falso café) son tóxicos en estado fresco (Freire et al. 2005; Martínez Crovetto 2012), pero en infusión son utilizados como medicina (Keller 2007, 2008; Martínez Crovetto 2012), y su madera blanda es buena iniciadora de fuego (Doumecq 2019).

A continuación, presentamos los rasgos y elementos diagnósticos resultantes del análisis del material fresco seguido de la caracterización de rasgos resultantes de distintas actividades de procesamiento:

Celtis tala Gillies ex Planch (Celtidaceae), "tala": posee frutos cuyo endocarpo contiene células pétreas rectangulares y circulares (Figura 3a) (Lema et al. 2010). Las hojas presentan cistolitos silicificados con valor diagnóstico que se desarrollan por debajo de pelos o células prominentes y pueden contener grandes cristales romboidales (Figura $3 b)$. Estas estructuras foliares fueron descriptas anteriormente por Fernández Honaine et al. (2005).

Jodina rhombifolia (Hook. \& Arn.) Reissek (Santalaceae), "sombra de toro": la semilla presenta células pétreas de formas irregulares (Figura 3c), campanuliformes, ovoides, subrectangulares y rectangulares, así como cristales en forma de prisma de base cuadrada (Lema et al. 2010).

Zea mays L. (Poaceae), "maíz": posee en sus semillas principalmente granos de almidón simples, poliédricos, con facetas de presión, tamaño variable de 2 a $35 \mu \mathrm{m}$; hilum céntrico en forma de $\mathrm{V}$, como un punto o línea; cruz central definida. Pueden aparecer en agregados de dos a más individuos (Winton y Winton 1932; Korstanje y Babot 2005; entre otros). 


\begin{tabular}{|c|c|c|c|c|c|}
\hline $\begin{array}{l}\text { Nombre registrado } \\
\text { en la fuente }\end{array}$ & $\begin{array}{l}\text { Taxonomía } \\
\text { actualizada }\end{array}$ & $\begin{array}{l}\text { Órgano usado } \\
\text { registrado }\end{array}$ & $\begin{array}{l}\text { Procesamiento } \\
\text { mencionado }\end{array}$ & $\begin{array}{c}\text { Práctica asociada } \\
\text { mencionada }\end{array}$ & Autor [año]/lugar \\
\hline $\begin{array}{l}\text { Abatí, millo, trigo } \\
\text { turco }\end{array}$ & Zea mays* & - & - & \begin{tabular}{|c|} 
Siembra, \\
alimento, \\
intercambio, robo, \\
recibimiento
\end{tabular} & $\begin{array}{c}\text { Ramírez [1528]/ Ríos } \\
\text { Paraná y Paraguay } \\
\text { Schmidel [1534-1554]/ Río } \\
\text { Paraguay }\end{array}$ \\
\hline $\begin{array}{l}\text { Cucurus, trigo turco, } \\
\text { maíz, maíz capia, } \\
\text { maíz morocho, } \\
\text { pisingallo, de colores }\end{array}$ & Zea mays* & Grano & $\begin{array}{l}\text { Fresco, tostado, } \\
\text { horneado, } \\
\text { hervido, mascado, } \\
\text { fermentado }\end{array}$ & $\begin{array}{c}\text { Siembra, alimento } \\
\text { y bebida }\end{array}$ & $\begin{array}{l}\text { Pauke [1749-1767]/ } \\
\text { Reducción San Javier, } \\
\text { Santa Fe (Argentina) }\end{array}$ \\
\hline Mandioca & Manihot sp* & Raíz & Molido & $\begin{array}{l}\text { Relaciones } \\
\text { sociales } \\
\text { (recibimiento) }\end{array}$ & $\begin{array}{c}\text { Ramírez [1528]/ } \\
\text { desembocadura Río } \\
\text { Paraguay }\end{array}$ \\
\hline Manteochade & Manihot sp* & Raíz & - & Alimento & $\begin{array}{c}\text { Schmidel [1534-1554]/ Río } \\
\text { Paraguay }\end{array}$ \\
\hline Mandeoch poere & Manihot sp* & Raíz & - & Bebida & $\begin{array}{c}\text { Schmidel [1534-1554]/ Río } \\
\text { Paraguay }\end{array}$ \\
\hline Mandeoch propie & Manihot sp* & Raíz & - & Alimento & $\begin{array}{c}\text { Schmidel [1534-1554] / Río } \\
\text { Paraguay }\end{array}$ \\
\hline Mandioca & Manihot sp* & Raíz & Hervido, secado & Siembra, alimento & $\begin{array}{l}\text { Pauke [1749-1767]/ } \\
\text { Reducción San Javier, } \\
\text { Santa Fe (Argentina) }\end{array}$ \\
\hline Manioc & Manihot sp* & Tubérculo & Molido & \begin{tabular}{|c|} 
Siembra, \\
alimento, bebida
\end{tabular} & $\begin{array}{l}\text { Levi-Strauss 1957/ } \\
\text { Amazonas brasilero }\end{array}$ \\
\hline & & & Fresco & Tóxico & \multirow{3}{*}{$\begin{array}{l}\text { Vargas Machuca (1892 } \\
\text { [1599])/Sudamérica }\end{array}$} \\
\hline Yuca brava & Manihot sp* & Raíz & $\begin{array}{l}\text { Fresco, rallado, } \\
\text { exprimido, seco, } \\
\text { molido }\end{array}$ & Alimento & \\
\hline Yuca dulce & Manihot sp* & Raíz & $\begin{array}{l}\text { Fresco, asado, } \\
\text { cocción }\end{array}$ & Alimento & \\
\hline \multirow[b]{2}{*}{$\begin{array}{l}\text { Manihot utilíssima, } \\
\text { mandioca, mandió }\end{array}$} & \multirow{5}{*}{ Manihot esculenta } & Raíz & Fresco & Tóxico & \multirow[b]{2}{*}{$\begin{array}{l}\text { Hieronymus 1882/ Gran } \\
\text { Chaco, Brasil }\end{array}$} \\
\hline & & - & $\begin{array}{l}\text { Cocción, tostado, } \\
\text { exprimido, secado, } \\
\text { fermentado }\end{array}$ & \begin{tabular}{|c|} 
Alimento, \\
medicina, \\
cosmético, bebida \\
alcohólica \\
\end{tabular} & \\
\hline \multirow{3}{*}{ manjy'o, mandioca } & & Raíz & $\begin{array}{l}\text { Descortezado, } \\
\text { enjuagado }\end{array}$ & Medicina & \multirow{3}{*}{$\begin{array}{l}\text { Keller }(2007,2008) / \\
\text { Misiones (Argentina) }\end{array}$} \\
\hline & & & - & Alimento & \\
\hline & & - & - & Cebo para caza & \\
\hline \multirow[b]{2}{*}{ Tala } & \multirow[b]{2}{*}{ Celtis tala } & Hoja & Infusión, fresco & Medicina & $\begin{array}{l}\text { Pochettino et al } 2016 . \\
\text { /Parque Costero del Sur } \\
\text { (Argentina) }\end{array}$ \\
\hline & & Fruto & Fresco & Alimento & $\begin{array}{l}\text { Rapoport et al. 2009/ } \\
\text { Sudam. Arenas 2003/Gran } \\
\text { Chaco (Argent.) Pochettino } \\
\text { et al. } 2016 \text { / Parque Costero } \\
\text { del Sur (Argentina) }\end{array}$ \\
\hline \multirow{2}{*}{ Sombra de toro } & \multirow{2}{*}{ Jodina rhombifolia } & \multirow{2}{*}{ Fruto } & Fresco & Alimento & \multirow{2}{*}{$\begin{array}{c}\text { Pochettino et al. 2016/Pque } \\
\text { Cost. del Sur (Argent.) } \\
\text { Hieronymus 1882/ } \\
\text { Córdoba (Argentina) }\end{array}$} \\
\hline & & & Extracto & Medicina & \\
\hline
\end{tabular}

Tabla 1. Información obtenida de fuentes documentales sobre especies que fueron incluidas en la colección de referencia. Se consigna su denominación según el nombre registrado en las fuentes, la identificación taxonómica asignada en las fuentes, el órgano utilizado, su procesamiento y prácticas asociadas, los autores y la región en la que hacen mención de las plantas. En los casos en que no se especifica en las fuentes analizadas y los datos brindados permiten hacerlo, señalamos una posible identificación taxonómica con un asterisco $\left(^{*}\right)$. 

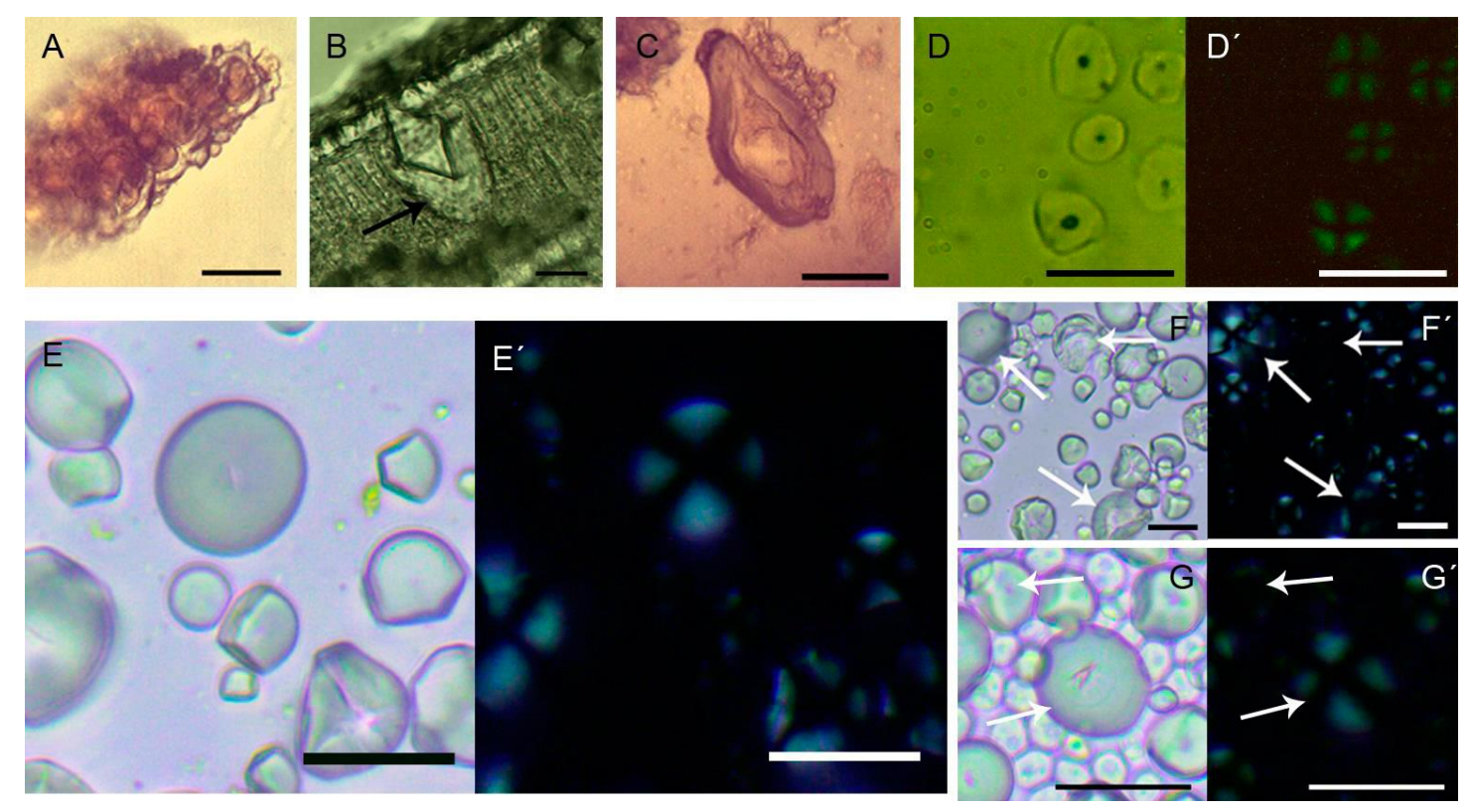

Figura 3. Resultados de la colección de referencia: A células pétreas de C. tala; B señalamos cistolito foliar de C. tala; C célula pétrea de J. rhombifolia; D-D' almidones de Z. mays bajo luz

normal (D) y polarizada (D'). E-E' almidones de M. esculenta con bajo luz normal (E) y polarizada (E'). F-G' almidones de M. esculenta luego de fermentación bajo luz normal (F y G) y polarizada ( $F^{\prime}$ y $\left.G^{\prime}\right)$, se señalan alteraciones resultados de fermentación (Fig. A y C: colección

LEBA; Fig. D: colección Laboratorio 129). Escala $20 \mu \mathrm{m}$.

Manihot esculenta Crantz. (Euphorbiaceae), "mandioca": los granos de almidón de estas raíces son simples o compuestos, en forma de campana, circulares $\mathrm{u}$ ovales. El tamaño varía entre 6 hasta $35 \mu \mathrm{m}$ de longitud. El hilum usualmente es central, y sobre él puede haber una fisura en forma de línea transversal, en Y o en cruz. La cruz de extinción generalmente es central con brazos rectos $\mathrm{y}$, en menor frecuencia, levemente excéntrica con brazos ondulados. Son comunes las facetas de presión en almidones truncados y en circulares. Granos grandes y pequeños pueden presentarse en agregados (Winton y Winton 1932; Pagán Jiménez 2015).

Estudios experimentales sugieren la aparición de distintos rasgos de valor diagnóstico (i.e. fisuras, grietas, perdida de birrefringencia, hidrólisis, entre otros) que dan cuenta de diferentes procesos culinarios, que varían en intensidad según los tiempos y los pasos involucrados, así como dependiendo de las plantas que intervienen. Los resultados de distintas formas de procesamiento de Zea mays fueron publicados por distintos autores (Babot 2003; Pagán Jiménez 2013; Petrucci y Lema 2016; entre otros). El tostado del maíz genera una combinación de partículas de carbón, y almidones intactos junto a otros con pérdida de birrefringencia, con deformación de la cruz de extinción, así como gelatinizados. Se observa en muchos almidones un centro oscuro en la zona del hilum, observable bajo luz normal y polarizada (Babot 2003; Petrucci y Lema 2016). Asimismo, el hervido genera un incremento en el tamaño de los granos y pérdida parcial o total de la birrefringencia. De este tipo de procesamiento también resulta un orificio circular sobre el hilum, que se presenta como un centro oscuro bajo luz normal y polarizada (Petrucci y Lema 2016). 
En cuanto a los resultados del proceso de fermentación sobre raíces de $M$. esculenta, Chandler-Ezell y equipo (2006) registraron una combinación de almidones alterados, agregados y gelatinizados. La fermentación de harina de mandioca en nuestras experimentaciones generó además alteraciones en la cruz de extinción (Figura 3e), orificios en la zona del hilum que, en algunos casos resultó en granos de almidón con forma anular; pérdida de birrefringencia; fragmentaciones y fisuras pudiendo dividir los granos en dos o más partes. Observamos también alteraciones en el contorno y superficie en grado bajo, medio y alto asociado a una baja visibilidad al MO de los granos (Figura 3f).

\section{Material arqueológico}

El análisis de las muestras recuperadas en los fragmentos cerámicos de Las Marías nos permitió observar diversas estructuras vegetales, así como fragmentos de organismos procariotas y animales. En la Tabla 2 (ver Material Anexo) sintetizamos los resultados obtenidos en los dieciséis fragmentos analizados separados en tres grandes grupos: contenedores de procesamiento y/o consumo, contenedores de almacenaje e instrumentos de transferencia.

Contenedores de procesamiento y/o servicio

Todas las muestras recuperadas de este grupo morfo-funcional contenían microrrestos, ya sean vegetales como protistas y/o animales (Figura 4a). Las estructuras vegetales registradas incluyen fibras de distintos tipos: (1) con lumen ancho en relación a las paredes y extremos aguzados; (2) planas, con torsiones sobre su eje, lumen estrecho en relación a la pared y extremos aguzados; (3) con lumen estrecho en relación a las paredes anchas de extremos redondeados, con saliencias a modo de mamelones.

Hallamos fitolitos de Celtis tala y otros afines a distintos grupos vegetales (Danthonioides, Panicoides, Arecoides y Festucoides, ver Figura 4b). Estos elementos silíceos se encuentran tanto en la superficie interna como externa de los contenedores, pero cabe resaltar que fueron mayoritarios, en cantidad y tipos morfológicos, en los fragmentos cerámicos del grupo $\mathrm{M}$ (muestras control). Registramos distintos morfotipos de esclereidas, principalmente células pétreas o braquiesclereidas y macroesclereidas (Crang et al. 2018), algunas de las cuales corresponden a Celtis tala (Figura 4g) y Jodina rhombifolia (Figura 4h). Observamos también tricomas estrellados (Figura 4i) y un grano de polen (Figura 4j).

En seis contenedores hallamos granos de almidón, los cuales se recuperaron principalmente de las superficies internas. Observamos almidones poliédricos que denominamos Tipo 1 y asignamos a Zea mays. Algunos presentaron centros con proyecciones oscuras en la zona del hilum asociados a microcarbones (Figura 4k y 41'). Otro conjunto de almidones estuvo representado por granos simples, con forma de 
campana. Muchos de estos gránulos no polarizaban, o presentaban una cruz de extinción incompleta. Estos almidones, que resultaron indeterminados, los llamamos Tipo 2 (Figura 41 y $41^{\prime}$ ).

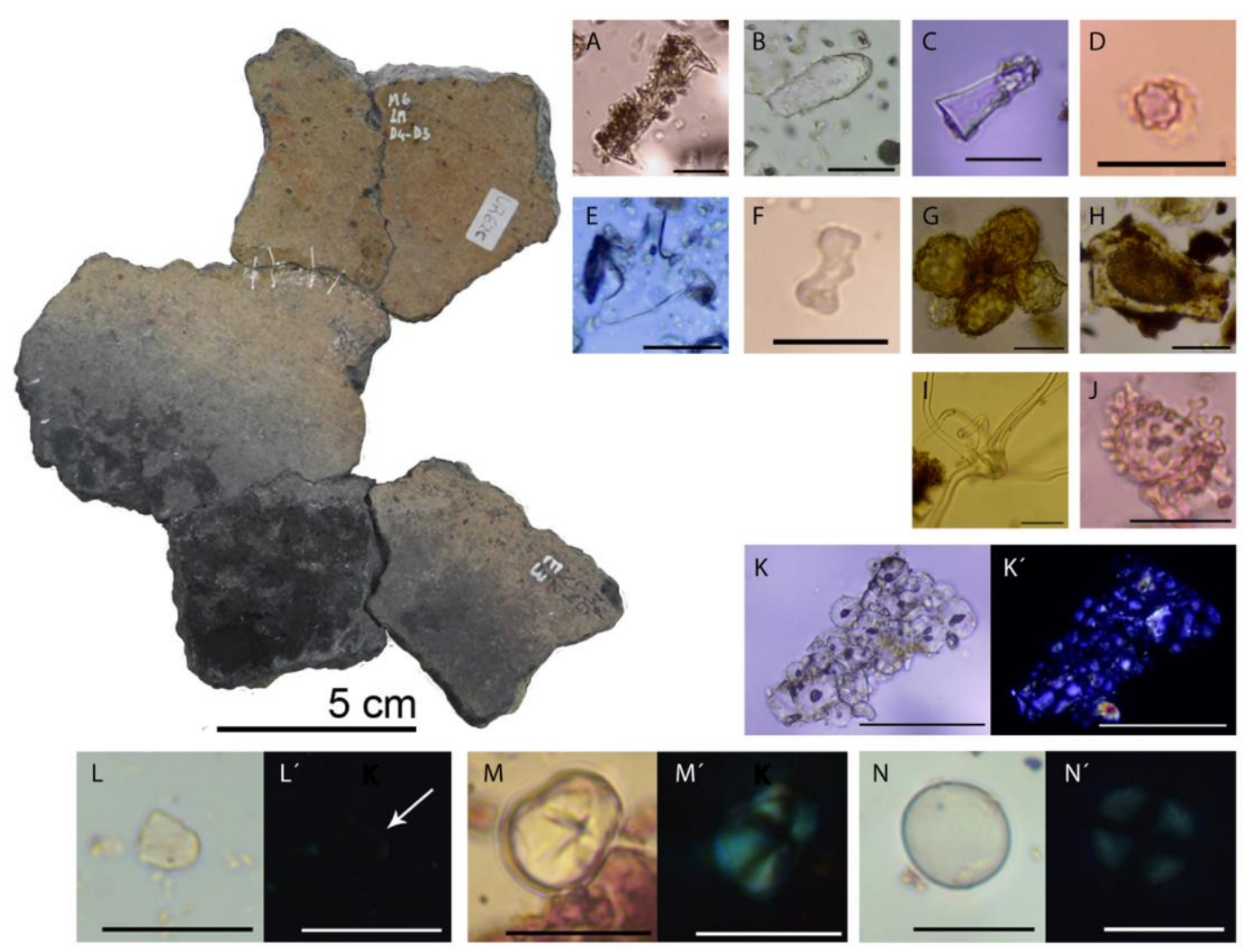

Figura 4. Izquierda-superior reconstrucción de base y cuerpo de un contenedor de procesamiento. A espícula de espongiario; B-F fitolitos: B cistolito de C. tala; C Danthonioide; D Arecoide; E Asteriforme; F halterio polilobulado; $\mathrm{G}$ células pétreas de $C$. tala; $\mathrm{H}$ célula pétrea de J. rhombifolia; I tricoma estrellado; J grano de polen; K almidones Tipo 1 de Z. mayz; L almidón Tipo 2 indeterminado y L' polarización, indicamos con una flecha el almidón con pérdida de birrefringencia; $\mathrm{M}$ almidón Tipo 3 aff. M. esculenta; $\mathrm{N}$ almidón Tipo 4 indeterminable. Escala de microrrestos $20 \mu \mathrm{m}$.

El tercer tipo de almidones que registramos consistió en granos simples, ovoides, con una fisura horizontal o en " $Y$ " en la zona del hilio y una cruz de extinción levemente excéntrica con signos de alteración, como brazos muy anchos y difusos (Figura $4 \mathrm{~m}$ y $4 \mathrm{~m}^{\prime}$ ). Los almidones Tipo 3 resultan afines a uno de los morfotipos característicos de Manihot esculenta. Además, el cuarto tipo de granos de almidón estuvo representado por elementos simples y esféricos a elipsoides, con cruz de extinción central (Figura $4 \mathrm{n}$ y $4 \mathrm{n}^{\prime}$ ). Los almidones Tipo 4 son ubicuos en el reino vegetal, por lo que son indeterminables.

Contenedores de almacenaje

En este tipo de contenedores registramos espículas de espongiarios (Figura 5a) y estomatocistes de algas Chrysophyceae en las superficies internas. Observamos también elementos indeterminables, como estructuras planas perforadas de posible 
origen animal (Figura 5b) y masas rojizas que en algunas ocasiones brillan bajo luz polarizada. El componente fitolítico se compone de elementos diagnósticos para Danthonioide, Panicoide y Festucoide (Figura 5c y 5e).

Observamos granos de almidón correspondientes a los Tipos 1 (Figura 5f y $\left.5 f^{\prime}\right), 3$ y 4 mencionados antes. Asimismo, registramos una nueva categoría de grano de almidón que denominamos Tipo 5. Se encuentran en forma individual o en agregados. La mayor parte presenta contornos y superficies muy degradados, asociados a fisuras que se proyectan desde el hilum. Las formas varían entre ovales, con tamaños similares al Tipo 3, y campanas, en su mayoría unidas por su base (Figura $5 g$ y $5 g^{\prime}$ ). La cruz de extinción levemente excéntrica por lo general está alterada, presentándose engrosada, difusa, y en algunos casos con múltiples brazos o brazos con torceduras (Figura $5 \mathrm{~h}$ y $5 \mathrm{~h}^{\prime}$ ). La birrefringencia bajo luz polarizada es media a baja y prima una baja visibilidad bajo luz normal, lo que dificultó su visualización. Considerados en conjunto, los Tipos 3 y 5 de almidones resultan afines a Manihot esculenta. Asimismo, los patrones de desgaste y alteración presentes en el Tipo 5 podrían corresponderse con actividades de fermentación observadas en la colección de referencia.
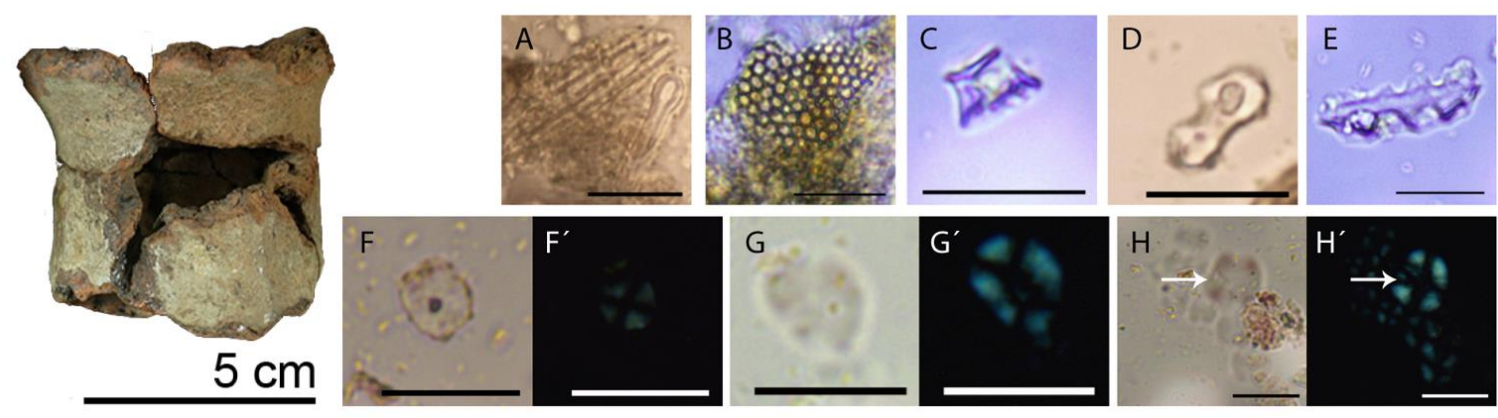

Figura 5. Izquierda parte superior de un contenedor de almacenaje; A espículas de espongiarios; B elemento indeterminable; C-E fitolitos: C Danthonoide, D Panicoide y E Festucoide; F-F' almidón Tipo 1 de Z. mays; G-H almidones Tipo 5 aff. M. esculenta: G-G' campanas, $\mathrm{H}^{-\mathrm{H}^{\prime}}$ señalamos cruz de extinción con brazos con modificaciones. Escala de microrrestos $20 \mu \mathrm{m}$.

Instrumentos de transferencia

Registramos espículas de espongiarios y estomatocistes de algas Chrysophyceae (Figura 6 A). En tanto el componente fitolítico, registramos morfologías no diagnósticas y otras de afinidad Danthonioide (Figura $6 \mathrm{~b}$ y $6 \mathrm{c}$ ). Observamos también almidones Tipo 1 (Zea mays), tanto agregados como individuales (Figura 6d y 6d'), así como otros que reúnen las características Tipo 3 y 5 (afines a M. esculenta; Figura 6e y 6f'); y Tipo 4. Por último, al igual que en las muestras cerámicas anteriores, registramos fragmentos de tejido vegetal y masas de color amarillento a rojizo, que en algunos casos son brillantes bajo luz polarizada y resultan indeterminables. 


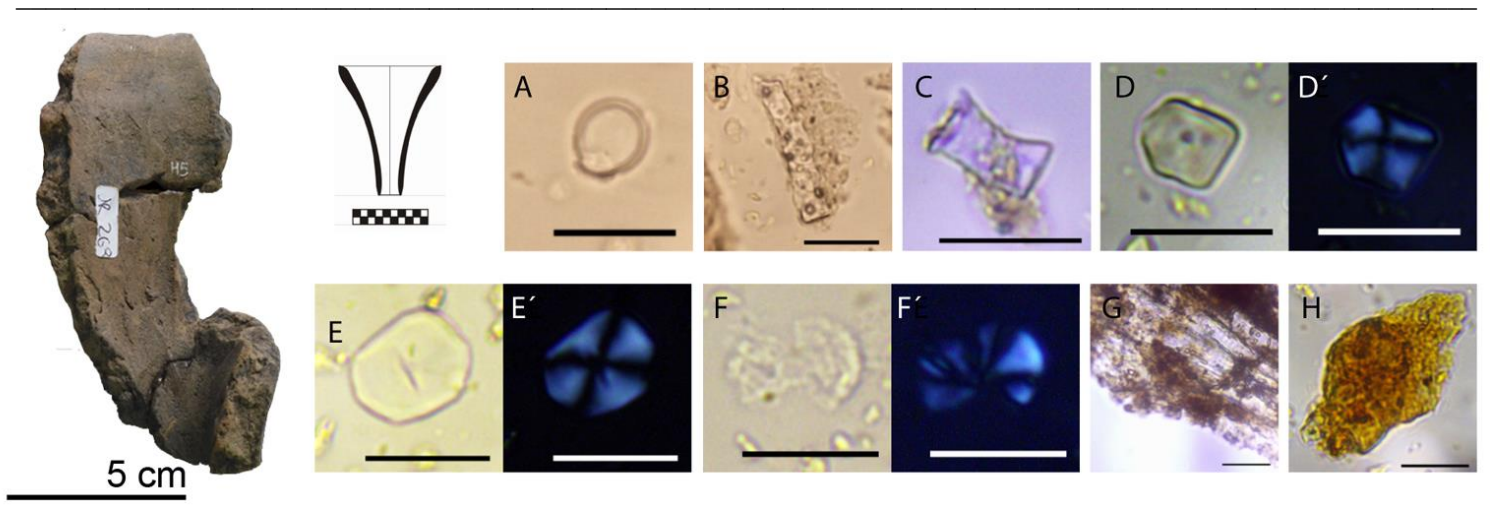

Figura 6. Izquierda fragmento de instrumento de transferencia y su reconstrucción digital; A estomatociste de alga Chrysophyceae, B fitolito elongado con superficie agujereada, C fitolito Danthonioide; D-D’almidón Tipo 1 de Z. mays, E-F' almidones aff. M. esculenta: E-E' Tipo 3; F-F' Tipo 5; G fragmento de tejido; $\mathrm{H}$ masa rojiza indeterminable. Escala de microrrestos $20 \mu \mathrm{m}$.

\section{Reflexiones y consideraciones finales}

El análisis realizado nos permitió observar la presencia de microrrestos vegetales en todas las muestras correspondientes al conjunto cerámico del sitio arqueológico Las Marías. Así también, pusimos a prueba estrategias metodológicas que resultaron satisfactorias para el caso de estudio. Registramos una diversidad de estructuras vegetales, correspondientes tanto a plantas silvestres como domesticadas con distintas formas de procesamiento.

Desde el punto de vista metodológico, la selección de muestras control nos permitió apreciar que aquellos fragmentos cerámicos que no fueron expuestos al lavado y acondicionamiento registran mayor cantidad y diversidad de estructuras vegetales. Estas estrategias nos permiten ajustar los protocolos de trabajo en laboratorio. Por otro lado, el uso de ácido láctico para decolorar costras quemadas adheridas a las superficies cerámicas facilitó la observación de elementos con buena preservación y su posterior determinación. La implementación de este procedimiento nos concedió subsanar estas dificultades y por ende constituyó un aporte metodológico significativo.

Para aproximarnos a los contextos de uso y prácticas asociadas por parte de los grupos que habitaron la zona articulamos la información procedente del análisis de microrrestos vegetales, con las fuentes documentales, la colección de referencia y los estudios cerámicos realizados. Destacamos que las fuentes documentales no sólo son herramientas para la formulación de hipótesis acerca del pasado, sino para realizar interpretaciones a partir de confrontar nuestras realidades con otras que nos permitan pensar en una variedad de situaciones o mundos posibles (Haber 2011). El relevamiento de crónicas y diarios del siglo XVI al XIX, así como de publicaciones etnobotánicas recientes, en asociación con el análisis paleoetnobotánico nos permitió incorporar a la colección de referencia plantas tanto silvestres de la región rioplatenseparanaense, como otras domesticadas de circulación macrorregional (como mandioca o 
maíz). Observamos la mención de distintos órganos almacenadores de almidón potencialmente útiles y asociados a diversas prácticas, ya sea alimenticias, medicinales o de intercambios sociales. En este sentido, cabe aclarar la importancia que reviste el análisis contextual de los documentos dado que permite plantear múltiples preguntas e interpretaciones. Tal es el caso del diario de Lopes de Sousa [1530-1532], donde la ausencia de menciones sobre vegetales domesticados vinculados a la descripción del modo de vida de poblaciones nativas se transforma en un elemento significativo para confrontar con otros relatos de la misma época en esa región. Una instancia para preguntarnos sobre los intereses que intervinieron, la abundancia estacional de recursos, el tipo de contacto que realizaron con poblaciones nativas, entre otros. Por otro lado, en fuentes documentales tempranas registramos para situaciones críticas el uso alimenticio de plantas posiblemente silvestres (Ramirez [1528]; Lopez de Sousa [1530-1532]; Schmidel [1599]). Por lo general, dichas plantas no se asocian explícitamente al conocimiento botánico de poblaciones nativas (con excepción de algunos casos en Schmidel [1599]), aunque no debemos descartarlo, ya que todas las expediciones tenían integrantes indígenas que hacían de intérpretes y guías.

El análisis arqueobotánico evidenció microrrestos vegetales en los tres tipos morfofuncionales, en algunos casos granos de almidón con rasgos que demuestran distintos tipos de procesamiento para su aprovechamiento. Los mismos también se recuperaron en bordes externos de las tres categorías alfareras: en cuellos curvos de contenedores de almacenaje, en extremos afinados de instrumentos de transferencia y en incisiones y superficies lisas cercanas al borde de contenedores de procesamiento y/o servicio. Consideramos que esto podría vincularse con los usos dados a los objetos y con la presencia de zonas que permitan la acumulación, entrampe y preservación de las estructuras vegetales. Asimismo, las espículas de espongiarios localizadas principalmente en superficies internas podrían relacionarse con la presencia de agua en las sustancias que se procesaron, almacenaron o manipularon.

Muchas estructuras registradas, entre ellas algunos granos de almidón con patrones diagnósticos, no resultaron compatibles con las muestras de referencia, de este modo los asignamos por el momento a la categoría "indeterminados". En consecuencia, consideramos que es indispensable seguir enriqueciendo la colección comparativa con materiales frescos y sometidos a distintos tipos de procesamientos, tanto para ajustar las determinaciones taxonómicas, como para aproximarnos de forma integral a los saberes botánicos de las poblaciones humanas del pasado.

Investigaciones previas de abordaje morfo-funcional del registro cerámico del sitio arqueológico Las Marías (ie. Paleo y Pérez Meroni 2008) encuentran en el presente trabajo una evidencia directa sobre los contenidos vegetales y tipos de procesamientos realizados sobre ellos. Podemos observar que los contenedores de almacenaje e instrumentos de transferencia se asocian al manejo de sustancias líquidas, ya sea fermentadas o maceradas, infusiones, entre otras; así como a la manipulación, almacenamiento o trasvaso de sustancias molidas como harinas. En tanto, los 
contenedores de procesamiento y/o servicio presentan evidencias de exposición al calor, que podrían corresponderse con la preparación de hervidos, asados, horneados o tostados.

Los resultados de las muestras analizadas revelan las primeras evidencias directas de la presencia y procesamiento de plantas domesticadas para la zona. Es importante destacar que estas se encuentran asociadas con otras de crecimiento espontáneo y que resultan características del bosque de tala, como Celtis tala y Jodina rhombifolia.

Los almidones de Zea mays se asocian con rasgos diagnósticos vinculados con la exposición al calor pudiéndose tratar de tostado, horneado o hervido. Estudios previos sobre restos orgánicos de tiestos cerámicos procedentes de este sitio realizados mediante cromatografía gaseosa con espectrómetro de masa, dieron como resultado que podrían corresponder a la cocción de peces o a maíz dulce (Paleo y Pérez Meroni, 2007; Pérez Meroni et al. 2010). En esa oportunidad, no se contaba con evidencias que avalen la utilización del maíz por parte de estos grupos. A partir del análisis de microrrestos aquí presentados podemos realizar una nueva lectura y sustentar con otra línea de evidencias la utilización de plantas domesticadas como Zea mays y posiblemente Manihhot esculenta. Los microrrestos asignados como afines a M. esculenta presentan características congruentes con prácticas de fermentación, maceración, u otras donde se produce la hidrólisis de los granos por acciones enzimáticas. Estas prácticas son compatibles con la confección de vinos y otras bebidas a partir de Manihot sp. registradas en crónicas tempranas (Schmidel [1534-1554]). En la zona de estudio crece Manihot grahamii Hook (o falso café). Este árbol no cuenta con estructuras de almacenamiento subterráneas como M. esculenta, y no hemos registrado el uso de sus raíces en fuentes documentales. Probablemente su vínculo con las poblaciones humanas se basó en el consumo de hojas con fines medicinales, la obtención de leña y sombra (Keller 2007, 2008; Martínez Crovetto 2012; Doumecq 2019).

En concordancia con el registro arqueológico, Celtis tala y Jodina rhombifolia presentan registros de uso en fuentes documentales tanto de sus frutos como hojas. Las células pétreas y cistolitos foliares descriptos en este trabajo se hallan en su totalidad en fragmentos de contenedores de procesamiento y/o servicio. Asimismo, se encuentran mayoritariamente en superficies internas y dentro de costras carbonizadas, así como en bordes o agujeros de suspensión o reparación, localizaciones que consideramos congruentes con el uso de cocción, fermentación, contención, derrame, servido o entrampe en pequeñas cavidades. Estas evidencias se incorporan a otros hallazgos arqueológicos en contextos de procesamiento de frutos de tala, tanto en cerámica como en instrumentos de molienda (Babot et al. 2007; Gonzalez y Frere 2009; Lema et al. 2010; Pérez Meroni et al. 2010), como así también en fogones (Aldazabal y Vazquez 2015). Oportunamente, al indagar sobre prácticas alimenticias asociadas a vegetales en el sitio Las Marías se propuso el uso de C. tala y J. rhombifolia, y se consideró que su procesamiento estaría vinculado a actividades de tostado y molienda implementadas para facilitar la cocción de frutos y semillas con tejidos ricos en almidón (Lema et al. 
2010; Pérez Meroni et al. 2010). Por otro lado, también existen registros antracológicos (Ghiani Echenique et al. 2020) y etnobotánicos (Doumecq 2019) del uso de estos árboles como combustible en la zona.

Los trabajos realizados hasta el momento evidencian que la diversidad de modos en que se relacionan las poblaciones locales con estas plantas de crecimiento espontáneo sugieren la presencia de distintas formas de manejo del entorno (Casas 2001). Este conjunto de actividades humanas diversas de control del ambiente tendientes a la preservación y proliferación de alguna/s población/es vegetal/es pueden considerarse como prácticas de cultivo en su sentido amplio (Casas 2001; Lema 2010). De esta forma, Jodina rhombifolia y Celtis tala podrían haber participado en actividades de protección, fomento y/o recolección que aseguren su presencia e involucramiento en distintas actividades (desde alimentos, medicinas, tinturas, hasta sombra, protección y reparo, creencias populares, entre otras). Las plantas domesticadas, aquellas que necesitan de la intervención humana para su supervivencia (sensu Ford 1979; Lema 2010), podrían haberse introducido a través de interacciones sociales con áreas vecinas (es el caso de Zea mays y posiblemente Manihot esculenta). Esta propuesta deberá ser complementada con análisis de sedimentos para esclarecer la probabilidad de actividades hortícolas en el seno de estas poblaciones ribereñas.

En este trabajo utilizamos categorías biológicas (como domesticada, cultivo, silvestre o espontánea) y económicas (como uso, recurso), y mantenemos la división entre seres humanos y plantas con fines prácticos y analíticos teniendo en cuenta sus limitaciones y connotaciones. Estas reflexiones nos permiten superar dicotomías como silvestre/domesticado y cazador-recolector/agricultor, y pensar una población con patrones de movilidad relativamente bajos que practique actividades de caza, pesca y recolección de una amplia gama de recursos, incluidas distintas técnicas de cultivo de vegetales, como parte de una diversidad de formas de manejo del entorno naturalcultural.

Agradecimientos: Esta contribución fue realizada con subsidios provenientes de UNLP y PICT, así como una beca doctoral CONICET. Agradecemos a lxs evaluadores anónimxs y a la Dra. Day Piraría por la lectura detenida y sus comentarios que enriquecieron este manuscrito, y a las Dras. Lema, Petrucci y Capparelli por facilitarnos el acceso digital a las colecciones de referencia del LEBA (FCNyM) y del Laboratorio 129 (Div. de Arqueología del Museo de Ciencias Naturales de La Plata) en tiempos de COVID-19. A dichos laboratorios pertenecen algunas imágenes correspondientes a la colección ro referencia que publicamos en este trabajo.

\section{Bibliografía citada}

\section{Aldazabal V. y F. Vázquez}

2015 El Registro Arqueobotánico del sitio El Divisadero Monte 6 (Partido de General Lavalle, Buenos Aires). Revista de Antropología del Museo de Entre Ríos 1 (2): 83-90 
Auge, M. A., D. Andreoni, M. S. García Lerena

2018 El uso de plantas como fumatorios en los partidos de Magdalena y Punta Indio (Buenos Aires): un análisis de microrrestos vegetales en pipas arqueológicas de la ribera rioplatense. Intersecciones en Antropología 19 (2): 145-157.

Babot M. P.

2003 Starch grain damage as an indicator of food processing. Phytolith and starch research in the Australian-Pacific-Asian regions: the state of the art (Eds. D. Hart \& L. Wallis), pp. 69-81. Pandanus Books, Canberra.

Babot M. P.; N. Mazzia y C. Bayón

2007 Procesamiento de recursos en la región pampeana bonaerense: aportes del instrumental de molienda de las localidades arqueológicas El Guanaco y Cerro La China En: Arqueología en las Pampas. Tomo II, C. Bayón, A. Pupio, M. I. González, N. Flegenheimer y M. Frère editores, pp. 635-657. Sociedad Argentina de Antropología, Buenos Aires.

Beovide, L.

2011 La presencia de cultígenos desde el quinto milenio en el registro arqueológico del curso medio platense: revisión y proyecciones. Avances y Perspectivas en la Arqueología del Nordeste (Eds. M.R. Feuillet Terzaghi, M.B. Colasurdo, J. Sartori y S. Escudero), pp. 155-173. Servicios Gráficos, Santo Tomé.

Beovide, L., y Campos, S.

2015 El Manejo Del Entorno Vegetal y Cultígenos (Zea Mays L.) en los Concheros Entre ca. 3.000 y 2.000 Años AP en la Cuenca Inferior del Río Santa Lucía, Uruguay. Cuadernos del Instituto Nacional de Antropología y Pensamiento LatinoamericanoSeries Especiales 2(4): 56-70.

Bonomo, M.; Aceituno, F. J.; Politis, G. G. \& Pochettino, M. L.

2011 Pre-Hispanic horticulture in the Paraná Delta (Argentina): archaeological and historical evidence. World Archaeology 43(4): 554-575.

Casas A.

2001 Silvicultura y domesticación de plantas en Mesoamérica. Plantas, cultura y -niedad. Estudio sobre la relación entre seres humanos y plantas en los albores del siglo XXI 76 ( $u$ s. B. Rendón Aguilar, S. Rebollar Domínguez, J. Caballero Nieto y M.A. Martínez Alfaro), pp. 123-158. Universidad Autónoma Metropolitana, México.

Chandler-Ezell, K., D. M. Pearsall and J. A. Zeidler

2006 Root and tuber phytoliths and starch grains document manioc (Manihot esculenta), arrowroot (Maranta arundinacea), and llerén (Calathea sp.) at the Real Alto site, Ecuador. Economic Botany 60(2): 103-120. 
Colobig, M. M. y Ottalagano, F. V.

2016 Estudio arqueobotánico de los residuos orgánicos adheridos en alfarerías prehispánicas de la cuenca del Paraná medio. Arqueología 22(1): 193-210.

Colobig, M. M.; Píccoli, C. V.; Barboza, M. C.

2016 Primera aproximación a la identificación de granos de almidón en tiestos del sitio Los Bananos (Goya, Corrientes, Argentina). Relaciones de la Sociedad Argentina de Antropología (41) 2: 431-440.

Cornero, S., y Rangone, L.

2015 Análisis arqueobotánicos en sitios de la entidad arqueológica Goya-Malabrigo ubicados en el centro-norte de Santa Fe. Anuario de Arqueología 7: 85-94.

Crang, R., Lyons-Sobaski, S., \& Wise, R.

2018 Plant anatomy: a concept-based approach to the structure of seed plants. Springer, Gewerbestrasse.

Day Pilaría, F. A.

2018 Gestión de los recursos faunísticos en sociedades cazadoras, recolectoras y pescadoras (Tesis Doctoral). Universidad Nacional de La Plata, La Plata.

Delucchi, G. y S. S. Torres Robles

2009 Plantas exóticas en el Parque Costero del Sur: Una categorización. Parque Costero del Sur. Naturaleza, conservación y patrimonio cultural (Ed. J. Athor), pp. 408-415. Fundación de Historia Natural Félix de Azara, Buenos Aires.

Doumecq M. B.

2019 Evaluación etnobotánica de los recursos vegetales combustibles actuales y potenciales de la Ribera Platense (Provincia de Buenos Aires, Argentina). Tesis doctoral. Universidad Nacional de La Plata, La Plata.

Fernández Honaine, M. F., Zucol, A., y Osterrieth, M.

2005 Biomineralizaciones de sílice en Celtis tala Planchon (Celtidaceae). Boletin de la Sociedad argentina de Botánica 40(3-4): 229-239.

Freire S. E.; A. M. Arambarri, N. D. Bayón, G. Sancho, E. Urtubey, C. Monti, M. C. Novoa y M. N. Colares

2005 Epidermal characteristics of toxic plants for cattle from the Salado river ba 77 (Buenos Aires, Argentina). Bol. Soc. Argent. Bot. 40 (3-4): 241-281.

Ford, R.

1979 Paleoethnobotany in American Archaeology. Advances in archaeological method and theory, Tomo 2 (Ed. M. Schiffer), pp. 285-336. Academic Press, Nueva York. 
Ghiani Echenique, N. M. y P. N. León

2014 Metodología 3D para la reconstrucción de formas cerámicas en contextos de cazadores-recolectores. Sitio Las Marías (partido de Magdalena, provincia de Buenos Aires). Entre pasados y presentes IV: Estudios contemporáneos en Ciencias Antropológicas (Eds. A. Castro Esnal, M. L. Funes, M. Grosso, N. Kuperszmit, A. Murgo y G. Romero), pp. 579-595. Asociación Amigos del Instituto Nacional de Antropología, Buenos Aires.

Ghiani Echenique N.; O. L. Sokol y M. Lozano

2017 Reconstrucción virtual. Un aporte a la arqueología en tres dimensiones. Cuadernos del Instituto Nacional de Antropología y Pensamiento Latinoamericano 4(4): 20-29.

Ghiani Echenique N.; C. Valencia y M. C. Paleo

2020 De buena madera. Análisis antracológico de restos leñosos carbonizados procedentes del sitio Los Tres Ombúes (partido de Punta Indio, provincia de Buenos Aires). Intersecciones en Antropología 21(1): 71-84.

González M. I. y M. M. Frère

2009 Talares y paisaje fluvial bonaerense: arqueología del río Salado. Intersecciones en Antropología 10: 249-265.

Haber, A.

2011 Animismo, relacionalidad, vida: perspectivas post-occidentales. Biografías de paisajes y seres: visiones desde Ia arqueología sudamericana (Coords. D. Hermo y L. Miotti), pp. 75-98. Editorial Brujas, Buenos Aires.

Hieronymus J.

1882 Plantae Fiaphoricae Florae argentinae. Kraft, Buenos Aires.

International Code of Starch Nomenclature (ICSN)

2011 The International Code for Starch Nomenclature. Recuperado de http://fossilfarm.org/index.html

Keller H. A.

2007 Unidades de vegetación y recursos florísticos en una aldea Mbya Guaraní de Misiones, Argentina. Kurtziana 33(1). Vol. Especial de etnobotánica: 175-191.

2008 Etnobotánica de comunidades guaranies de Misiones, Argentina; valoración de la vegetación como fuente de recursos. Tesis Doctoral. Universidad Nacional del Nordeste, Corrientes.

Korstanje, A. y M. P. Babot

2005 Microfossils characterization from South Andean economic plants. Plants, people and places (Eds. M. Madella y D. Zurro), pp. 41-72. Oxbow books, Londres. 
Lema, V.

2010 Procesos de domesticación vegetal en el pasado prehispánico del noroeste argentino: estudio de las prácticas más allá de los orígenes. Relaciones-Sociedad Argentina de Antropología 35: 121-142.

Lema, V., M. L. Pochettino, M. Pueblas, M. C. Paleo Y M. Pérez Meroni

2010 La etnobotánica como herramienta interpretativa en arqueología: prácticas de recolección en el Holoceno tardío del litoral bonaerense (Argentina). Tradiciones y transformaciones en etnobotánica (Eds. M. L. Pochettino, A. Ladio y P. Arenas), pp. 38-43. Universidad Nacional de Jujuy, San Salvador de Jujuy.

Lema, V., D. Andreoni, A., G. Ortiz, R. Spano, M. Quesada Y F. Zorzi

2015 Protocolos y avances en el estudio de residuos de pipas arqueológicas de Argentina. Aportes para el entendimiento de metodologías actuales y prácticas pasadas. Estudios Atacameños 51: 77-97.

Lévi-Strauss, C.

1952 The use of wild plants in tropical South America. Economic Botany 6 (3): 252-270.

Lopes de Sousa, P.

1927 [1530-1532] Diario da Navegaçao de Pero Lopes de Sousa, Vol 1. Río de Janeiro, Brasil: Typographia Leuzinger.

Martínez Crovetto R. N.

2012 Estudios etnobotánicos V. Nombres de plantas y su utilidad según los mbya guaraní de Misiones, Argentina. Bonplandia 21 (2): 109-133.

Nacuzzi, L. R.

2002 Leyendo entre líneas: una eterna duda acerca de las certezas. Historia y estilos de trabajo de campo en Argentina (Coomps. S. Visacovsky y R. Guber), pp. 229-262. Editorial Antropofagia, Buenos Aires.

Neumann K.; C. A. E. Strömberg; B. Terry; R. M. Albert; L. Vrydaghs \& L. Scott Cummings: International Committee for Phytolith Taxonomy

2019 International Code for Phytolith Nomenclature (ICPN) 2.0. Annals of Botany 124 (2): 189-199.

Pagán Jiménez, J. R.: Proyecto Prometeo (SENESCYT )

2013 Evaluando algunos mecanismos de conservación/degradación en almidones modernos por medio de ensayos y experimentos controlados que replican ciertas formas antiguas de procesamiento y cocción de órganos almidonosos. Ensayo de laboratorio (reporte preliminar): Elaboración de dos tipos de chicha de maíz: chicha fermentada con saliva y otra con levadura. Recuperado de https://www.researchgate.net/publication/275344048_Evaluando_algunos_mecanis 
mos_de_conservaciondegradacion_en_almidones_modernos_por_medio_de_ensayos y_experimentos_controlados_que_replican_ciertas_formas_antiguas_de_procesamient o_y_coccion_de_organos

Pagán-Jiménez, J. R.

2015 Almidones. Guía de material comparativo moderno del ecuador para los estudios paleoetnobotánicos en el Neotrópico. ASPHA, Buenos Aires, Argentina.

Paleo, M. C.; Páez, M. y M. Pérez Meroni

2002 Condiciones ambientales y ocupación humana durante el Holoceno tardío en el litoral fluvial bonaerense). Del Mar a los Salitrales. Diez mil años de Historia Pampeana en el Umbral del Tercer Milenio (Eds. M. Berón, D. Mazanti y F. Olivera, pp. 365-376. Laboratorio de Arqueología y Sociedad Argentina de Antropología, Mar del Plata.

Paleo, M. C.; Perez Meroni, M.

2004 Problemáticas vinculadas a las estrategias de subsistencia de la localidad arqueológica Barrio San Clemente. La región Pampeana. Su pasado arqueológico (Eds. C. Gradín y F. Oliva), pp: 311-319. Laborde, Rosario.

2005-2006 Dimensión social de la tecnología cerámica en sociedades cazadorasrecolectoras. Rev. do Museu de Arqueologia e Etnologia, (15-16): 73-85.

2007 Primeros resultados del sitio "Las Marías", Partido de Magdalena. Provincia de Buenos Aires. Arqueología Argentina en los inicios de un nuevo siglo (Eds. F. Oliva, N. de Grandis y J. Rodríguez), Tomo 1, pp. 275-286. Laborde, Rosario.

2008 Relación forma-función de un conjunto alfarero del partido de Magdalena, provincia de Buenos Aires: una aproximación metodológica.). Problemática de la Arqueología Contemporánea (Eds. A. Austral y M. Tamagnini), pp. 219-226. Universidad Nacional de Río Cuarto, Río Cuarto.

Patterer, N. I.; E. Passeggi y A. F. Zucol

2011 Análisis fitolíticos de suelos del sudoeste de la Provincia de Entre Ríos (Argentina) como una herramienta para comprender sus procesos pedológicos. Rev. Mex. Cs. Geol. 28 (1): 132-146

Paucke F.

2010 [1749-1767] Hacia allá y para acá. Ministerio de Innovación y Cultura de la Provincia de Santa Fe, Santa Fe.

Pérez Meroni, M., Paleo, M. C., Pochettino, M. L., y Lema, V. S.

2010 Procesamiento y consumo de vegetales por grupos cazadores-recolectores del Holoceno tardío, en los partidos de Magdalena y Punta Indio, provincia de Buenos Aires. Mamül Mapu: Pasado y presente desde la arqueología pampeana, Tomo 1 (Eds. M. Berón, L. Luna, M. Bonomo, C. Montalvo, C. Aranda y M. Carrera-Aizpitarte), pp. 87102 Libros del Espinillo, Ayacucho. 
Petrucci, N. S. y Lema, V.S.

2016 Primeras aproximaciones a la identificación de técnicas de procesamiento en carporrestos de Zea mays L.: detección de granos hervidos en restos arqueobotánicos secos y carbonizados. Intersecciones en Antropología, 17: 291-302.

Pochettino, M. L., y Lema, V. S.

2008 La variable tiempo en la caracterización del conocimiento botánico tradicional. Darwiniana, 46(2): 227-239.

Pochettino M. L., M. C. Paleo, N. Ghiani Echenique, M. B. Doumecq y J. A. Hurrell 2016 La construcción del paisaje del litoral rioplatense 1. Las plantas y sus usos como patrimonio del Parque Costero del Sur. Servicop, La Plata.

Ramírez L.

2007 [1528] Carta de Luis Ramirez a su padre- Puerto de San Salvador, 10 de Julio de 1528. Carta de Luis Ramírez a su padre desde el Brasil (1528): orígenes de lo 'real maravilloso' en el Cono Sur (Ed. F. Maura). Lemir, (16): 1-63. Recuperado de http:/ / parnaseo.uv.es/Lemir/Textos/Ramirez.pdf

Rapoport, E. H., A. Marzocca y B. S. Drausal

2009 Malezas comestibles del Cono Sur y otras partes del planeta. Instituto Nacional de Tecnología Agropecuaria, San Carlos de Bariloche.

Reichert E. T.

1913 The diferentiation and specificity of starches in relation to genera, species, etc. Stereochemistry applied to protoplasmic processes and products, and as a strictly scientific basis for the clasification of plants and animals. Tomo $1 \mathrm{y} 2$. The Carnegie Institution of Washington, Washington.

Schmidel, U.

1903 [1599] Viaje al Río de la Plata (1534- 1554). Cabaut y Cía. editores, Buenos Aires.

Vargas Machuca de D. B.

1892 [1599] Milicia y Descripción de las Idias. Vol. 1. Librería de Victoriano Suarez Preciados, Madrid.

Winton, A. \& K. B. Winton

1932 The structure and composition of foods. Tomo 1 y 2. John Willey \& Sons, New York. 
Zucol, A. F., y M. Bonomo

2008 Estudios Arqueobotánicos del Sitio Nutria Mansa 1 (Partido de General Alvarado, Provincia de Buenos Aires): II. Análisis Fitolíticos Comparativos de Artefactos de Molienda. Matices interdisciplinarios en estudios fitolíticos y de otros microfósiles (Eds. M. A. Korstanje y M. P. Babot), pp. 163-172. BAR International Series S.; Oxford.

Zucol, A. F., y D. Loponte

2008 Análisis comparativo metodológico y estudio de la abundancia fitolítica en tártaro de dientes humanos de sitios arqueológicos de la provincia de Buenos Aires, Argentina. Matices interdisciplinarios en estudios fitolíticos y de otros microfósiles (Eds. M. A. Korstanje y M. P. Babot), pp. 39-45. BAR International Series S.; Oxford. 